Article

\title{
Nonlinear Predictive Control of a Hydropower System Model
}

\section{Runfan Zhang, Diyi Chen and Xiaoyi Ma *}

Institute of Water Resources and Hydropower Research, Northwest A\&F University, Yangling 712100, China; E-Mails: runfanzhang@outlook.com (R.Z.); diyichen@nwsuaf.edu.cn (D.C.)

* Author to whom correspondence should be addressed; E-Mail: ieee307@163.com; Tel.:+ 86-29-8708-2860.

Academic Editor: Benjamin L. Ruddell

Received: 15 June 2015 / Accepted: 26 August 2015 / Published: 1 September 2015

\begin{abstract}
A six-dimensional nonlinear hydropower system controlled by a nonlinear predictive control method is presented in this paper. In terms of the nonlinear predictive control method; the performance index with terminal penalty function is selected. A simple method to find an appropriate terminal penalty function is introduced and its effectiveness is proved. The input-to-state-stability of the controlled system is proved by using the Lyapunov function. Subsequently a six-dimensional model of the hydropower system is presented in the paper. Different with other hydropower system models; the above model includes the hydro-turbine system; the penstock system; the generator system; and the hydraulic servo system accurately describing the operational process of a hydropower plant. Furthermore, the numerical experiments show that the six-dimensional nonlinear hydropower system controlled by the method is stable. In addition, the numerical experiment also illustrates that the nonlinear predictive control method enjoys great advantages over a traditional control method in nonlinear systems. Finally, a strategy to combine the nonlinear predictive control method with other methods is proposed to further facilitate the application of the nonlinear predictive control method into practice.
\end{abstract}

Keywords: nonlinear predictive control; hydropower system; Lyapunov function; control method; stability 


\section{Introduction}

Hydropower, as a low-cost, zero-pollution and renewable energy, has been developed since the twentieth century [1]. Thanks to modern technologies, hydro-turbines are becoming bigger and bigger. As a result, many hydropower plants with great capacity are being built around the world to generate electricity to resolve the serious energy problem. The system of such a powerful hydropower plant including penstock systems, water turbines, generators, regulators and loads, is so complex that it is difficult to control [2]. However, the stability of a hydropower system plays an important role in the stability of the whole power system and the plants themselves [3]. Thus, the issue of the control and stability of hydropower systems enjoys great popularity among researchers. For example, in [4], a new adjustment method of PID governors was proposed for hydropower plants with long penstocks to control the power frequency. A nonlinear hydropower plant system was controlled by a fuzzy control method in [2]. A micro-hydro power plant model with a smaller, lighter, more robust and more efficient higher-speed turbine was built in [5], and a control scheme was also proposed. In [6], an accurate new dynamical model for a cascaded hydropower plant, a complex nonlinear system involving interacting input and output nonlinear parameters, nonlinear flow rates, and nonlinear dynamical hydraulic heads was developed. The primary control system and stability analysis of a hydropower plant was discussed in [7], and controllers, including PI, PID, and PI-PD, were studied. In [8], a control method based on integrating the entropy and mean value of the tracking error with the constraints was proposed for hydro-turbine speed governors.

The control problem relates to making the system reach the expect points or states. At present, there are an increasing number of control theories, and parts of them even have been applied in practice for many years such as PID control [8,9], adaptive control [10-13], and feedback control [14-16] and so on. On the contrary, many remarkable state-of-the-art control methods, which enjoy a variety of advantages compared to traditional control methods, are being developed. For instance, a discrete sliding mode controller coupled with a Kalman filter was designed in [17-19] to control the combustion phasing in real-time model based on the control method of Homogenous Charge Compression Ignition. However, the sliding model control has the chattering problem when the controllers switch to the other states, so a no-chattering sliding model control method was proposed in [20]. A kind of switched uncertain nonlinear system was controlled by an adaptive fuzzy tracking control method in [21]. In [22], multi-zone buildings were identified by an artificial neural network and controlled by predictive control techniques. The robust $H_{\infty}$ finite-time control method for a discrete system was discussed in [23]. Compared with the abovementioned methods, nonlinear predictive control theory is easier to apply in practice, and can be combined with traditional methods, which takes advantages of both traditional methods and predictive control as shown later in this paper.

Predictive control is an optimization problem. Using the discrete model of a system, the system's future states with the controllers are predicted. Then, based on the predicted values, appropriate controllers are found to minimize the performance index [24]. This control method is called Model Predictive Control (MPC), and if the controlled system is a nonlinear system, it will be Nonlinear Model Predictive Control (NMPC). The predicted distance is called the predictive length. In fact, only the first set of predicted controllers is selected to control the system to obtain the states of the next step. This relates to the reduction of the errors $[24,25]$. In the performance index, the terminal penalty function, the 
distance between states and the expected states, and the distance between the controllers and the expected controllers, which generally are zeros, are considered [24]. The concept of predictive control was originated in 1967 by Lee and Markus [24,25]. However, not only the hardware but also the software couldn't achieve very rapidly their computing mission in Lee's and Markus' paper. Thus, predictive control was only a concept in that time. Thanks to modern technologies, predictive control started enjoying popularity in the late 1970s. Later, in 1982 Chen and Shaw first brought the concept of nonlinear predictive control in continuous time proved by the Lyapunov function [26]. Since then the predictive control method has aroused attention. For example, many remarkable studies have been done in different fields [27-34], such as large-scale optimization problems [29], hybrid electric vehicles [30], and the shell heavy oil fractionator benchmark control problem [31], and so on.

Motivated by what has been discussed above, we provide a simple method to select the performance index for a discrete time model proved by the Lyapunov function. The proof process is so intelligible that experts and technicians can understand and apply this method without too much effort. A six-dimensional complex nonlinear hydropower plant system is controlled based on the proposed method. In addition, we supply instructions for technicians in other fields or other hydropower plants to apply this method. Moreover, the strategy is also introduced to combine the NMPC with other traditional control methods.

The remaining parts of this paper are organized as follows: in Section 2, the principal concepts including a simply method to select performance index and the effectiveness of the proposed method are presented. A nonlinear hydropower plant model is introduced in Section 3. The main results of the controlled hydropower system are illustrated in Section 4. In Section 5, we discuss the effectiveness of the NMPC and how to apply this method in the other situations, involving the combination of the NMPC with other control methods.

\section{Nonlinear Predictive Control}

\subsection{System Model}

The continuous system can be described as:

$$
\dot{\mathbf{x}}=\mathbf{F}(\mathbf{x}, \mathbf{u}),
$$

where $\mathbf{x} \in R^{n}, \mathbf{u} \in R^{m}$, the constraints of $\mathbf{x}$ and $\mathbf{u}$ are $\mathbf{x} \in X, \mathbf{u} \in U$, respectively; and $\mathbf{F}(\cdot)$ is a function in $X \times U$ and Lipschitz continuous.

Assume that Equation (1) has an equilibrium point $\left(\mathbf{x}_{\mathbf{0}}, \mathbf{u}_{\mathbf{0}}\right)=(\mathbf{0 , 0})$. If $\mathbf{F}(\cdot)$ does not follow the assumption, we should move the equilibrium point $\left(\mathbf{x}_{\mathbf{0}}, \mathbf{u}_{\mathbf{0}}\right)$ to $(\mathbf{0 , 0})$ through transformation of coordinates. Equation (1) can be discretized as:

$$
\mathbf{x}_{k+1}=\mathbf{f}\left(\mathbf{x}_{k}, \mathbf{u}_{k}\right),
$$

where $\mathbf{f}(\cdot)$ is a function in $X \times U$, and is also Lipschitz continuous.

Note that if the system is a discrete system, Equation (2) is able to directly describe the system. 


\subsection{Nonlinear Predictive Control Theory}

The concept of predictive control is that the system can be predicted by Equation (2). Based on the state vector $\mathbf{x}_{k}$ with time $k$, several future state vectors of the system are able to be predicted with the input parameters $\mathbf{u}_{k}$ as:

$$
\mathbf{x}_{k+i+1}=\mathbf{f}\left(\mathbf{x}_{k+i}, \overline{\mathbf{u}}_{k+i}\right),(i=0, \cdots, N),
$$

where $\mathbf{x}_{k+}$ are the predicted values based on the states $\mathbf{x}_{k}$, and $\overline{\mathbf{u}}_{k+i}$ are the predictive controllers based on the states $\mathbf{x}_{k}$ calculated later, $N$ is the predict length.

The controllers $\overline{\mathbf{u}}_{k+i}$ are supposed to make the performance index $J_{k}$ be minimum. The $J_{k}$ is always defined as:

$$
J_{k}=\sum_{j=0}^{N-1} L\left(\mathbf{x}_{k+j}, \overline{\mathbf{u}}_{k+j}\right)+V\left(\mathbf{x}_{k+N}\right)
$$

In this case, the predictive control issue is to solve the following optimization problem:

$$
\overline{\mathbf{u}}_{k+j} \mid \underset{\min }{\mid J_{k}}=\sum_{j=0}^{N-1} L\left(\mathbf{x}_{k+j}, \overline{\mathbf{u}}_{k+j}\right)+V\left(\mathbf{x}_{k+N}\right), \quad \mathbf{x} \in X, \mathbf{u} \in U, \text { and } \mathbf{x}_{k+N} \in \Omega_{\alpha},
$$

where $L(\cdot)$ should follow Assumption 1, $V(\cdot)$ is the terminal penalty function subjected to Assumption 2, and $\Omega_{\alpha}$ is the terminal region defined later. However, after the sets of controllers $\overline{\mathbf{u}}_{k+i}$ are calculated, in order to reduce the errors caused by the controllers, only the first set controllers:

$$
\mathbf{u}_{k}^{*}=\overline{\mathbf{u}}_{k}
$$

are working in one step at the system, which is:

$$
\mathbf{x}_{k+1}=\mathbf{f}\left(\mathbf{x}_{k}, \mathbf{u}_{k}^{*}\right) \text {. }
$$

\section{Assumption 1}

(1). $L(0,0)=0$;

(2). $L(\cdot)$ is Lipschitz continuous;

(3). There exists a positive parameter $\delta$ satisfying $L(\mathbf{x}, \mathbf{u}) \geq \delta\|(\mathbf{x}, \mathbf{u})\|$.

\section{Assumption 2}

There exist a local controller $u_{v}$ satisfying

(1). $V\left(\mathbf{f}\left(\mathbf{x}, \mathbf{u}_{v}\right)\right)-V(\mathbf{x}) \leq-L\left(\mathbf{x}, \mathbf{u}_{v}\right)$;

(2). $V(\cdot)$ is Lipschitz continuous.

In order to determine an appropriate terminal penalty function $V(\cdot)$ to make the system be stable or asymptotic stable, the following efforts are needed. We linearize the system (1) by Jacobian method at the origin as:

$$
\dot{\mathbf{x}}=A \mathbf{x}+B \mathbf{u}
$$


where $A=\left.\frac{\partial \mathbf{F}}{\partial \mathbf{x}}\right|_{(\mathbf{x}, \mathbf{u})=(\mathbf{0}, \mathbf{0})}$ and $B=\left.\frac{\partial \mathbf{F}}{\partial \mathbf{u}}\right|_{(\mathbf{x}, \mathbf{u})=(\mathbf{0}, \mathbf{0})}$. If the system (8) can be stabilized, the linear feedback controllers $\mathbf{u}_{f}=K \mathbf{x}$ can be found to stabilize the system (7). Then the system is:

$$
\dot{\mathbf{x}}=G \mathbf{x}
$$

where $G=A+B K$.

\section{Theorem 1}

If the function $L(\cdot)$ is defined as $L(\mathbf{x}, \mathbf{u})=\mathbf{x}^{T} Q \mathbf{x}+\mathbf{u}^{T} R \mathbf{u}$, where $Q$ and $R$ are positive symmetric matrixes, respectively. The terminal penalty function $V(\cdot)$ will be $V(\mathbf{x})=\mathbf{x}^{T} P \mathbf{x}$, where $P$ is a positive-definite matrix solved by the Lyapunov equation as:

$$
(G+\kappa I)^{T} P+P(G+\kappa I)=-\left(Q+K^{T} R K\right), \quad \kappa<-\lambda_{\max }^{\lambda}(G)
$$

\section{Proof}

Defining $Q^{*}=Q+K^{T} R K$, the Lyapunov equation is:

$$
(G+\kappa I)^{T} P+P(G+\kappa I)=-Q^{*} .
$$

It is easy to know that $Q^{*}$ is positive-definite and symmetric. As mentioned above, if the system (9) is asymptotic stable, the real parts of all eigenvalues of $G$ will be negative, i.e.,

$$
\operatorname{Re}(\lambda(G))<0 \text {. }
$$

Since $\kappa<-\underset{\max }{\lambda}(G)$, it is simple to know that the real parts of all eigenvalues of $(G+\kappa I)$ are negative. Thus, in accordance with the solvability condition of Lyapunov equation, $P$ solved by Equation (11) is positive-definite and symmetric.

For a discrete system, the differential can be defined as the difference value between two steps for a per unit length. Thus:

$$
\frac{d}{d t} \mathbf{x}^{T} P \mathbf{x}=V\left(\mathbf{x}_{k+1}\right)-V\left(\mathbf{x}_{k}\right)=V\left(f\left(\mathbf{x}, \mathbf{u}_{v}\right)\right)-V(\mathbf{x})
$$

where $\mathbf{u}_{v}=K \mathbf{x}$ are feedback controllers. Substituting $\mathbf{u}_{v}$ for $\mathbf{u}$ in the system (1), we have:

$$
\dot{\mathbf{x}}=\mathbf{F}\left(\mathbf{x}, \mathbf{u}_{v}\right)=\mathbf{F}(\mathbf{x}, K \mathbf{x}) .
$$

Then:

$$
\begin{aligned}
\frac{d}{d t} \mathbf{x}^{T} P \mathbf{x} & =\left(\frac{d \mathbf{x}}{d t}\right)^{T} P \mathbf{x}+\mathbf{x}^{T} P\left(\frac{d \mathbf{x}}{d t}\right) \\
& =(G \mathbf{x}+\varphi(\mathbf{x}))^{T} P \mathbf{x}+\mathbf{x}^{T} P(G \mathbf{x}+\varphi(\mathbf{x})) \\
& =\mathbf{x}^{T} G^{T} P \mathbf{x}+\mathbf{x}^{T} P G \mathbf{x}+\varphi^{T}(\mathbf{x}) P \mathbf{x}+\mathbf{x}^{T} P \boldsymbol{\varphi}(\mathbf{x}) \\
& =\mathbf{x}^{T}\left(G^{T} P+P G\right) \mathbf{x}+2 \mathbf{x}^{T} P \boldsymbol{\varphi}(\mathbf{x})
\end{aligned}
$$

where $\varphi(\mathbf{x})=\mathbf{F}(\mathbf{x}, K \mathbf{x})-G \mathbf{x}$. 
The function $\mathbf{x}^{T} P \varphi(\mathbf{x})$ is bounded and its bound is:

$$
\mathbf{x}^{T} P \boldsymbol{\varphi}(\mathbf{x}) \leq\left\|\mathbf{x}^{T} P\right\| \cdot\|\boldsymbol{\varphi}(\mathbf{x})\| \leq\|P\| \cdot L_{\varphi}\|\mathbf{x}\|_{P}^{2} \leq \frac{\|p\| \cdot L_{\varphi}}{\lambda(P)}\|\mathbf{x}\|_{P}^{2},
$$

where $L_{\varphi}=\sup \left\{\frac{\|\boldsymbol{\varphi}(\mathbf{x})\|}{\|\mathbf{x}\|} \mid \mathbf{x} \in \Omega_{\alpha}, \mathbf{x} \neq \mathbf{0}\right\}$, and selecting an appropriate $\alpha$ satisfying $0<\alpha \leq \alpha_{1}$ enables:

$$
L_{\varphi} \leq \frac{\kappa \underset{\min }{\lambda}(P)}{\|P\|}
$$

By substituting (17) into (16), one obtains:

$$
\mathbf{x}^{T} P \varphi(\mathbf{x}) \leq \kappa \mathbf{x}^{T} P \mathbf{x} .
$$

Then, substituting (18) into (15), we have:

$$
\frac{d}{d t} \mathbf{x}^{T} P \mathbf{x} \leq \mathbf{x}^{T}\left((G+\kappa I)^{T} P+P(G+\kappa I)\right) \mathbf{x} .
$$

Substituting the inequality (19) into Equation (11) leads to:

$$
\begin{aligned}
\frac{d}{d t} \mathbf{x}^{T} P \mathbf{x} & \leq-\mathbf{x}^{T} Q^{*} \mathbf{x} \\
& \leq-\mathbf{x}^{T}\left(Q+K^{T} R K\right) \mathbf{x} \\
& \leq-\left(\mathbf{x}^{T} Q \mathbf{x}+\mathbf{x}^{T} K^{T} R K \mathbf{x}\right) . \\
& \leq-\left(\mathbf{x}^{T} Q \mathbf{x}+\mathbf{u}^{T} R \mathbf{u}\right) \\
& \leq-L(\mathbf{x}, \mathbf{u})
\end{aligned}
$$

From Equation (13) and Equation (20), it is rational and reasonable for us to obtain the equation in Assumption 2.1. This is the end of the proof. The terminal region $\Omega_{\alpha}$ is $\left\{\Omega_{\alpha} \mid \mathbf{x}^{T} P \mathbf{x} \leq \alpha\right\}$.

\section{Definition 1}

If there is a system as:

$$
\mathbf{x}_{k+1}=\mathbf{H}\left(\mathbf{x}_{k}\right) .
$$

If there exists a $K_{\infty}$ function $\boldsymbol{\beta}(\cdot)$ making $\mathbf{x}_{k}$ subject to $\left\|\mathbf{x}_{k}\right\| \leq \boldsymbol{\beta}\left(\mathbf{x}_{0}\right)$, the system will be Input-to-State-Stability (ISS) [35].

\section{Definition 2}

If there exists $K_{\infty}$ functions $\chi_{1}(\cdot), \chi_{2}(\cdot), \chi_{3}(\cdot)$ enabling:

$$
\chi_{1}(\|\mathbf{x}\|) \leq \Phi(\mathbf{x}) \leq \chi_{2}(\|\mathbf{x}\|), \boldsymbol{\Phi}(\mathbf{H}(\mathbf{x}))-\Phi(\mathbf{x}) \leq-\chi_{3}(\|\mathbf{x}\|) .
$$

then the continuous function $\boldsymbol{\Phi}(\cdot)$ is an ISS Lyapunov function of system (21). 


\section{Deduction 1}

If there exist an ISS Lyapunov function of system (21), the system will be an ISS system.

\section{Theorem 2}

If the predictive controllers are calculated in accordance with Equations (5) and (7), the system (2) will be an ISS system.

Proof

The predicted values based on $\mathbf{x}_{k-1}$ are denoted as $\tilde{\mathbf{x}}_{k-1+i}$, the corresponding predictive controllers are $\tilde{\mathbf{u}}_{k-1+i}$, and the performance index is $\tilde{J}$; similarly, the predicted values based on $\mathbf{x}_{k}$ are $\hat{\mathbf{x}}_{k+i}$, the predictive controllers are $\hat{\mathbf{u}}_{k+i}$, and the performance index is $\hat{J}$.

Then, (in the following process, Assumption 2.1 is applied):

$$
\begin{aligned}
\Delta J=\hat{J}-\tilde{J}= & \sum_{i=0}^{N-1}\left(\hat{\mathbf{x}}_{k+i}^{T} Q \hat{\mathbf{x}}_{k+i}+\hat{\mathbf{u}}_{k+i}^{T} R \hat{\mathbf{u}}_{k+i}\right)+\hat{\mathbf{x}}_{N}^{T} P \hat{\mathbf{x}}_{N} \\
& -\sum_{i=0}^{N-1}\left(\tilde{\mathbf{x}}_{k+i-1}^{T} Q \tilde{\mathbf{x}}_{k+i-1}+\tilde{\mathbf{u}}_{k+i-1}^{T} R \tilde{\mathbf{u}}_{k+i-1}\right)-\tilde{\mathbf{x}}_{N-1}^{T} P \tilde{\mathbf{x}}_{N-1} \\
= & \sum_{i=0}^{N-2}\left(\hat{\mathbf{x}}_{k+i}^{T} Q \hat{\mathbf{x}}_{k+i}+\hat{\mathbf{u}}_{k+i}^{T} R \hat{\mathbf{u}}_{k+i}-\tilde{\mathbf{x}}_{k+i}^{T} Q \tilde{\mathbf{x}}_{k+i}-\tilde{\mathbf{u}}_{k+i}^{T} R \tilde{\mathbf{u}}_{k+i}\right) \\
& +\hat{\mathbf{x}}_{N-1}^{T} Q \hat{\mathbf{x}}_{N-1}+\hat{\mathbf{u}}_{N-1}^{T} R \hat{\mathbf{u}}_{N-1}-\tilde{\mathbf{x}}_{k-1}^{T} Q \tilde{\mathbf{x}}_{k-1}-\tilde{\mathbf{u}}_{k-1}^{T} R \tilde{\mathbf{u}}_{k-1} \\
& +\hat{\mathbf{x}}_{N}^{T} P \hat{\mathbf{x}}_{N}-\tilde{\mathbf{x}}_{N-1}^{T} P \tilde{\mathbf{x}}_{N-1} \\
\leq & -\tilde{\mathbf{x}}_{k-1}^{T} Q \tilde{\mathbf{x}}_{k-1}-\tilde{\mathbf{u}}_{k-1}^{T} R \tilde{\mathbf{u}}_{k-1}
\end{aligned}
$$

In accordance with Assumption 1.3 and Equation (23), we have:

$$
\Delta J \leq-L\left(\mathbf{x}_{k-1}, \mathbf{u}_{k-1}\right) \leq-\delta\left\|\left(\mathbf{x}_{k-1}, \mathbf{u}_{k-1}\right)\right\|
$$

From Definition 2 and Equation (24), the performance index $J$ is the Lyapunov function of the controlled system (2) based on the control method in Equation (5). Therefore, according to Deduction 1, the system (2) with predictive controllers is an ISS system. This is the end of the proof.

The predictive control procedures are separated into two main parts: the offline part and online part. In the offline part process, the model of predictive control including positive-definite matrixes $Q$, and $R$, the feedback controller $K$, and the positive-definite matrix $P$ serving for solving the terminal region can be calculated. Since the controller $\mathbf{u}_{k}$ relates to the latest states $\mathbf{x}_{k}$ in each step, we can sensibly obtain the controllers in this part:

$$
\mathbf{u}_{k}=\mathbf{v}\left(\mathbf{x}_{k}\right)
$$

In terms of the online part, the states of system (2) will be iterated. Because the predictive controller (25) has been solved offline, we just need to substitute the numeric values $\mathbf{x}_{k}$ into Equation (25) to get the values of the controllers in each step. As a result, system (2) can respond rapidly. This is very important for two reasons. On one hand, if the controllers fail to be calculated in a short time, the states of the system will change to $\mathbf{x}_{k+\tau}$, where $\tau$ is the time for computers to calculate the controllers. The previous controllers (25) cannot enable system (2) to be stable in $\mathbf{x}_{k+\tau}$ with a large $\tau$. On the other 
hand, the primary mission of the controller is to maintain a stable system in some special situations. For example, in an electrical system, when the states of the system dramatically change caused by the cut out faulty lines because of the bad weather or the other reasons, the controllers are needed to stabilize the system with the rapid altered states; the nearby connected power plants of the lines should also be controlled in a short time. In these cases, the controllers are supposed to respond as fast as possible to make sure that the system operates stably and safely. Therefore, that is the reason why it is of significance for a system to enjoy rapid responsive controllers.

\section{The Model of a Hydropower Plant}

In this part, the nonlinear model of a hydropower plant is presented. The hydro-turbine, the penstock system, the generator system, and the hydraulic servo system are considered in this model [36]. The relationship between the deviation of the incremental torque and output power can be expressed as:

$$
P_{m}=m_{t}+\omega,
$$

where $m_{t}$ is the deviation of the incremental torque; $\omega$ is the variation of the speed of the generator. A typical diagram of the hydro-turbine and penstock system is shown in Figure 1 [37].

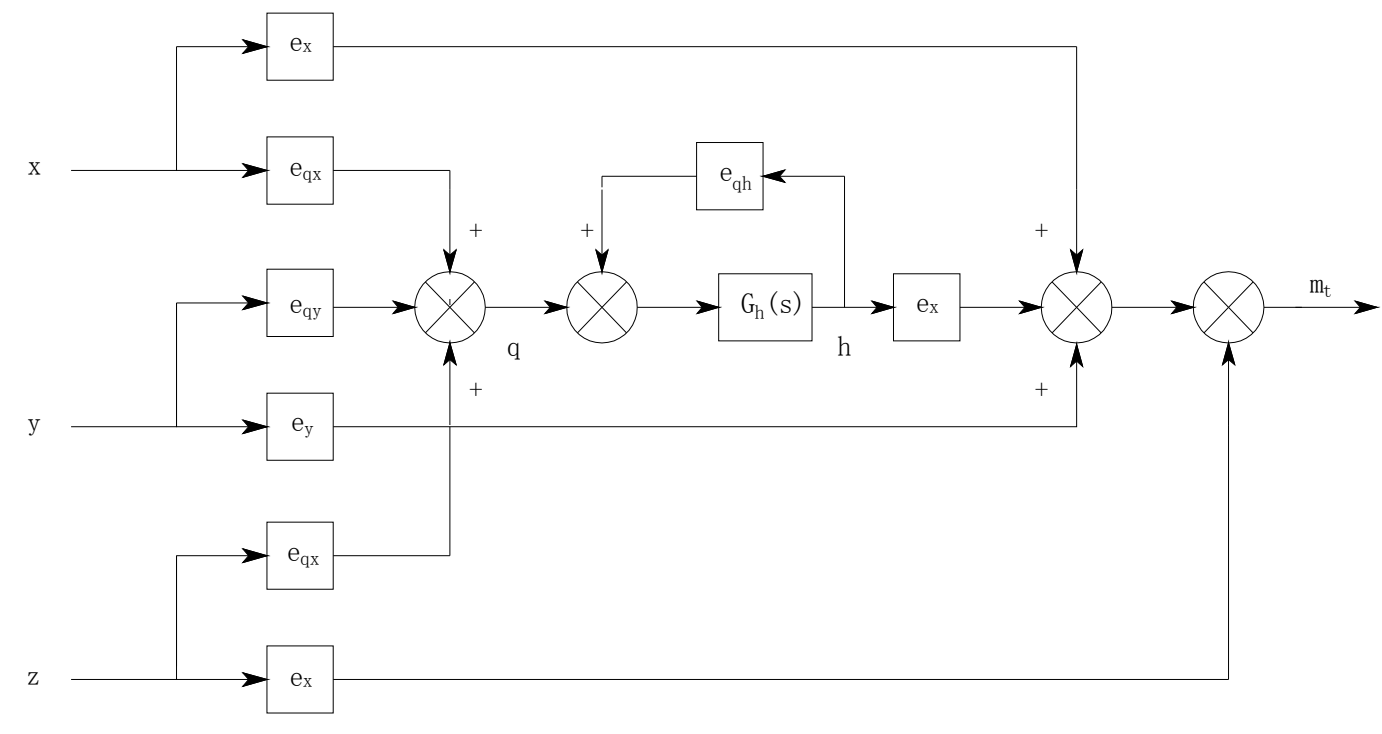

Figure 1. The linear model of the hydro-turbine and penstock system.

We suppose that the cross-sectional area of the penstock is constant. Thus the transfer function of the hydro-turbine and penstock system can be written as:

$$
G_{t}(s)=e_{y} \frac{1+e G_{h}(s)}{1-e_{q h} G_{h}(s)},
$$

where $e_{q h}$ is the first-order partial derivative value of flow rate with respect to water head; $e$ is the intermediate variable; $e_{y}$ is the first-order partial derivative value of torque with respect to wicket gate; $G_{h}(s)$ is the water hammer transfer function, described by:

$$
G_{h}(s)=\frac{H_{A}(s)}{Q_{A}(s)}=-2 h_{w} t h\left(0.5 T_{r} s\right)
$$


where $h_{w}$ is the characteristic coefficient of the penstock; $T_{r}$ is the length of the phase of the water hammer wave. From [37], the transfer function of the penstock system can be rewritten as:

$$
G_{h}(s)=-2 h_{w} \frac{\frac{1}{48} T_{r}^{3} s^{3}+\frac{1}{2} T_{r} s}{\frac{1}{8} T_{r}^{3} s^{2}+1} .
$$

Substituting Equation (29) into Equation (27), the transfer function between the incremental deviation of the guide vane opening $y$ and the deviation of the incremental torque $m_{t}$ can be rewritten as:

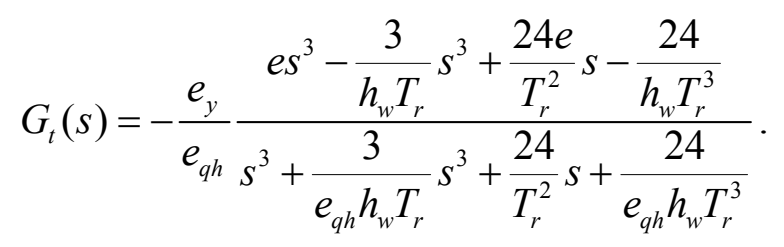

From Equation (30), the state space equations of the hydro-turbine and penstock system can be described as:

$$
\left\{\begin{array}{l}
\dot{x}_{1}=x_{2} \\
\dot{x}_{2}=x_{3} \\
\dot{x}_{3}=-a_{0} x_{1}-a_{1} x_{2}-a_{2} x_{3}+y
\end{array}\right.
$$

and:

$$
m_{t}=b_{3} y+\left(b_{0}-a_{0} b_{3}\right) x_{1}+\left(b_{1}-a_{1} b_{3}\right) x_{2}+\left(b_{2}-a_{2} b_{3}\right) x_{3}
$$

where $x_{1}, x_{2}$ and $x_{3}$ are state variables, $a_{0}=\frac{24}{e_{q h} h_{w} T_{r}^{3}}, a_{1}=\frac{24}{T_{r}^{2}}, a_{2}=\frac{3}{e_{q h} h_{w} T_{r}}, b_{0}=\frac{24 e_{y}}{e_{q h} h_{w} T_{r}^{3}}$, $b_{1}=-\frac{24 e e_{y}}{e_{q h} T_{r}^{2}}, b_{2}=\frac{3 e_{y}}{e_{q h} h_{w} T_{r}}$ and $b_{3}=-\frac{e e_{y}}{e_{q h}}$.

A second-order mathematical model of the generator is:

$$
\left\{\begin{array}{l}
\dot{\delta}=\omega_{0} \omega \\
\dot{\omega}=\frac{1}{T_{a b}}\left[m_{t}-m_{e}-D \omega\right]+u_{\omega}
\end{array}\right.
$$

where $u_{\omega}$ is predictive controller designed later.

If the influence of the rotor speed on the torque is added to the damping factor, the torque of the electrical load and the terminal active power are equal to each other, i.e.,

$$
m_{e}=P_{e} \text {. }
$$

For the generator, the terminal active power can be described as:

$$
P_{e}=\frac{E_{q}^{\prime} V_{s}}{x_{d \Sigma}^{\prime}} \sin \delta+\frac{V_{s}^{2}}{2} \frac{x_{d \Sigma}^{\prime}-x_{q \Sigma}}{x_{d \Sigma}^{\prime} x_{q \Sigma}} \sin 2 \delta
$$

and: 


$$
\left\{\begin{array}{l}
x_{d \Sigma}^{\prime}=x_{d}^{\prime}+x_{T}+\frac{1}{2} x_{L} \\
x_{q \Sigma}=x_{q}+x_{T}+\frac{1}{2} x_{L}
\end{array},\right.
$$

where $E_{q}^{\prime}$ is the transient internal voltage of the armature; $V_{s}$ is the bus voltage at infinity; $x_{d}^{\prime}$ is the direct axis transient reactance; $x_{q}$ is the quartered axis reactance; $x_{T}$ is the short circuit reactance of the transformer; $x_{L}$ is the reactance of the electric transmission line.

The dynamic characteristics of a hydraulic servo system [38] can be obtained as:

$$
T_{y} \frac{d y}{d t}+y=u_{y}
$$

where $y$ is the incremental deviation of the guide vane opening.

From Equation (26) to Equation (37), combining every part of the governing system into an organic whole as:

$$
\left\{\begin{array}{l}
\dot{x}_{1}=x_{2} \\
\dot{x}_{2}=x_{3} \\
\dot{x}_{3}=-a_{0} x_{1}-a_{1} x_{2}-a_{2} x_{3}+y \\
\dot{\delta}=\omega_{0} \omega \\
\dot{\omega}=\frac{1}{T_{a b}}\left[m_{t}-\frac{E_{q}^{\prime} V_{s}}{x_{d \Sigma}^{\prime}} \sin \delta-\frac{V_{s}^{2}}{2} \frac{x_{d \Sigma}^{\prime}-x_{q \Sigma}}{x_{d \Sigma}^{\prime} x_{q \Sigma}} \sin 2 \delta-D \omega\right]+u_{\omega} \\
\dot{y}=\frac{1}{T_{y}}\left(-y+u_{y}\right)
\end{array}\right.
$$

where $u_{y}$ and $u_{\omega}$ are selected as predictive controllers. The parameters in this paper are $w_{0}=314$, $T_{a b}=8.0, \mathrm{D}=0.5, E_{q}^{\prime}=1.35, x_{d \Sigma}^{\prime}=1.15, x_{q \Sigma}^{\prime}=1.474, T_{y}=0.1, V_{s}=1.0, e_{q h}=0.5$, $e_{y}=1.0, e=0.7, T_{r}=1.0, h_{w}=2.0, r=0, a_{0}=24, a_{2}=3, b_{0}=24, b_{1}=33.6, \quad b_{2}=3$, and $b_{3}=-1.4$, respectively.

\section{Main Results}

The numerical experiments of the nonlinear predictive control of the six-dimensional hydropower plant are presented in this part. In Equation (38), $\mathbf{u}=\left(u_{y}, u_{\omega}\right)^{T}$ are predictive controllers designed in accordance with Equations (5) and (6) later. The following positive-definite matrixes are given as:

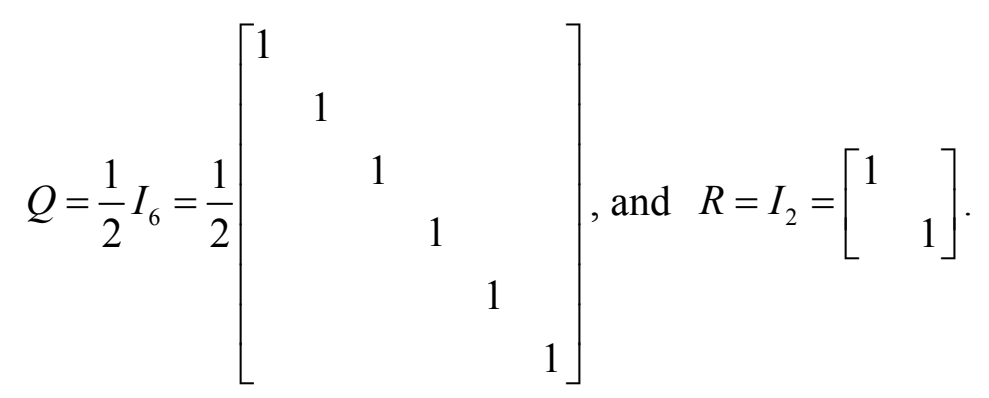

From what has been mentioned in Equation (8), we have: 


$$
A=\left[\begin{array}{cccccc}
0 & 1 & 0 & 0 & 0 & 0 \\
0 & 0 & 1 & 0 & 0 & 0 \\
-24 & -24 & -3 & 0 & 0 & 1 \\
0 & 0 & 0 & 0 & 314 & 0 \\
5.95 & 0 & 0.9 & -0.1228 & -0.0625 & -0.175 \\
0 & 0 & 0 & 0 & 0 & -10
\end{array}\right], \text { and } B=\left[\begin{array}{cc}
0 & 0 \\
0 & 0 \\
0 & 0 \\
0 & 0 \\
1 & 0 \\
0 & 10
\end{array}\right]
$$

The feedback gain $G$ in Equation (9) is $G=-\frac{1}{2} I_{4}$, so it is easy to get the feedback controllers $K$ by solving the equation $G=A+B K$, and $K$ is:

$$
K=\left[\begin{array}{cccccc}
-5.95 & 0 & -0.9 & 0.1228 & -0.4375 & 0.175 \\
0 & 0 & 0 & 0 & 0 & 0.95
\end{array}\right]
$$

By solving the Lyapunov Equation (11) when $\kappa=0.45<0.5=-\underset{\max }{\lambda}(G)$, the terminal penalty matrix $P$ is:

$$
P=\left[\begin{array}{cccccc}
359 & 0 & 53.55 & -7.309 & 26.03 & -10.41 \\
0 & 5 & 0 & 0 & 0 & 0 \\
53.5 & 0 & 13.1 & -1.106 & 3.938 & -1.575 \\
-7.309 & 0 & -1.106 & 5.151 & -0.5375 & 0.215 \\
26.03 & 0 & 3.938 & -0.5375 & 6.914 & -0.7656 \\
-10.41 & 0 & -1.575 & 0.215 & -0.7656 & 14.33
\end{array}\right]
$$

Now, after discretizing the system (38), and calculating the predictive controllers based on the above parameters, Equations (5) and (6), the states of the $\delta$ and $\omega$ of the controlled system, shown in Figure 2, can be obtained by iterating Equation (7).

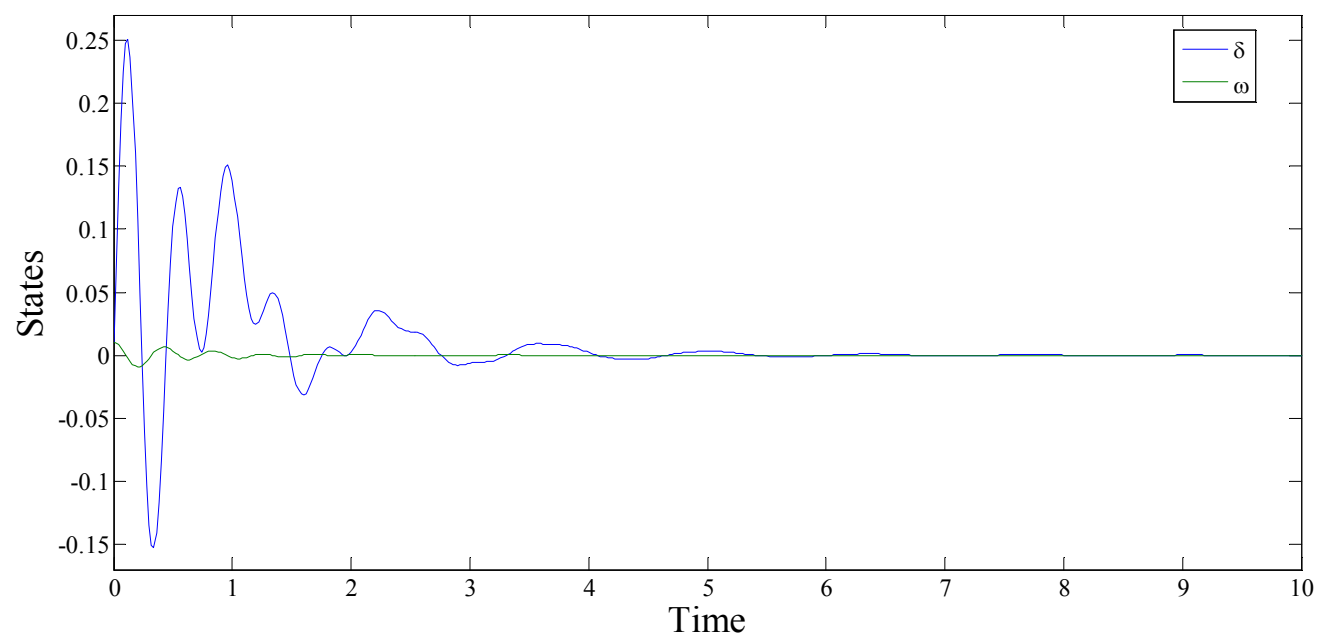

Figure 2. The response of the system (38) with NMPC controllers.

The predictive controllers $u_{y}$ and $u_{\omega}$ are shown in Figures 3 and 4, respectively. 


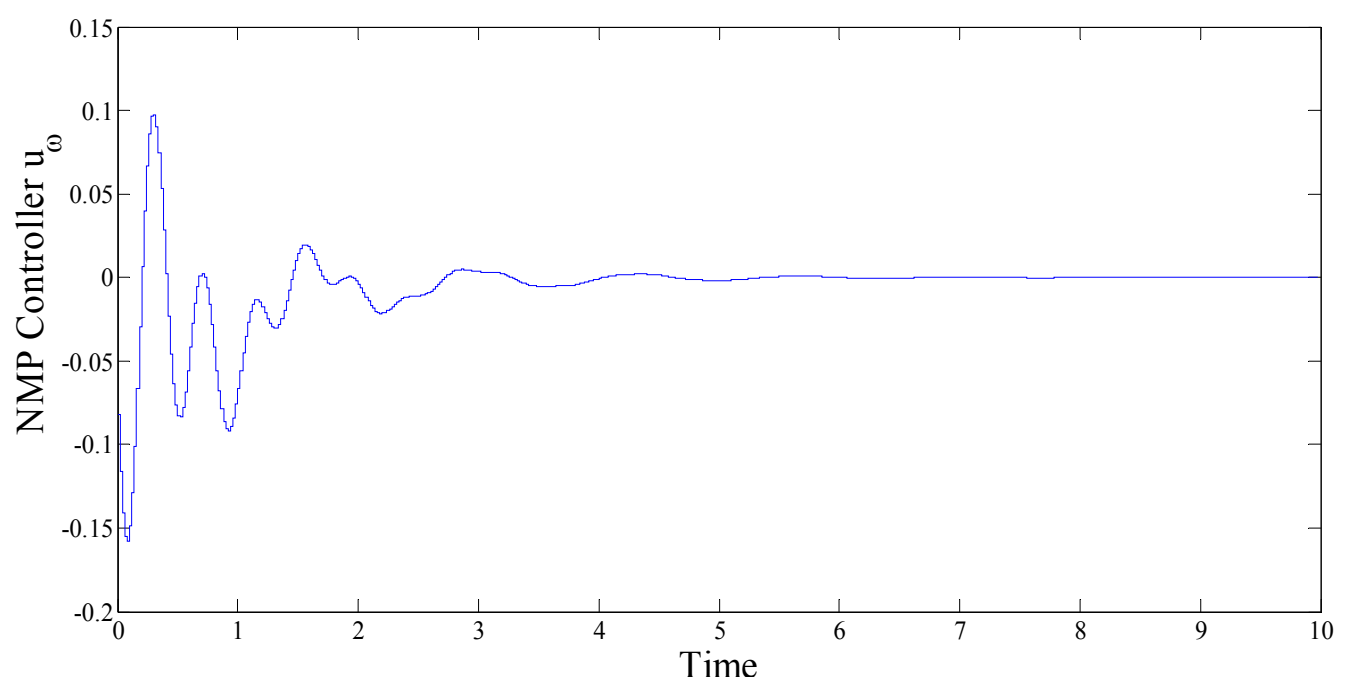

Figure 3. The NMPC controller $u_{\omega}$ of system (38).

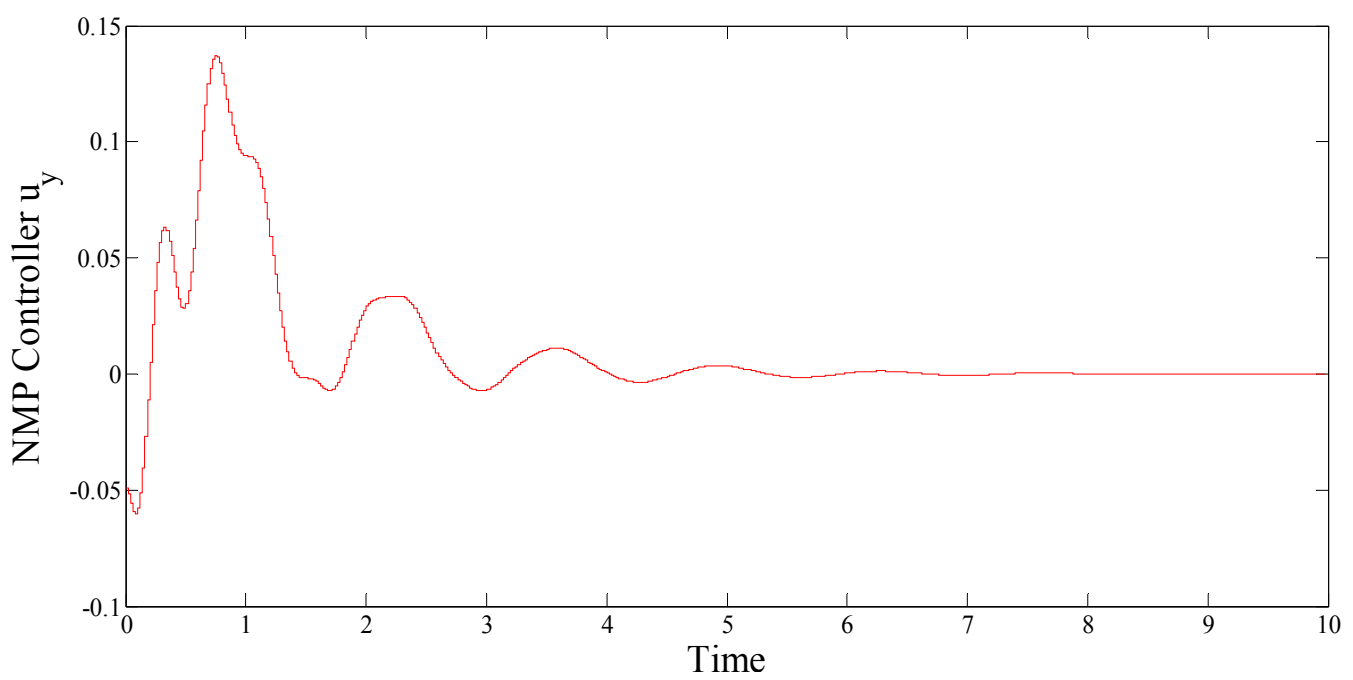

Figure 4. The NMPC controller $u_{y}$ of system (38).

From Figures 2-4, it is clear that the states of the hydropower system and the controllers are stable. The computational advantages of the NMPC method used in this paper are significant because the controllers were calculated offline. It should mention that our NMPC algorithm is separated into two parts. Obviously, only the elapsed CPU time of the online part relates to the fast response performance. The computational statistics of the offline part and online part are shown as follows.

Table 1 shows that the elapsed CPU time of the online part involves the entire horizon. We cannot ignore that the CPU used in the experiment is an Intel(R) Core(TM) i5 CPU, M $520 @ 2.40 \mathrm{GHz}$ (4 CPUs), the original mobile version of the Intel(R) Core(TM) i-series. If people use the latest CPU, they will obtain a better performance.

Table 1. The computational statistics of the NMPC method.

\begin{tabular}{cc}
\hline \multicolumn{2}{c}{ Elapsed CPU Time (ms) } \\
\hline Offline part & 5370 \\
Online part & 11,569 \\
\hline
\end{tabular}


The values of the performance index are presented as follows. In Table 2, the performance index is presented with gaps of 10 steps. It would be a long sheet if we were to present all the performance indexes here. The performance indexes have little difference when the step is bigger than 191. Thus, we omit the remaining indexes here. From Table 2, we can rationally and reasonably conclude that the performance index tends to zero as the step increases, which means the system is asymptotic stable, corresponding with the theoretic knowledge and the numerical experiment.

Table 2. The performance metrics of the NMPC method.

\begin{tabular}{cccc}
\hline Number of Step & Performance Index & Number of Step & Performance Index \\
\hline 1 & 0.117856 & 101 & 0.0022 \\
11 & 0.039339 & 111 & 0.012638 \\
21 & 0.030683 & 121 & 0.006103 \\
31 & 0.050499 & 131 & 0.001474 \\
41 & 0.077429 & 141 & 0.000687 \\
51 & 0.119509 & 151 & 0.001066 \\
61 & 0.016413 & 161 & 0.00026 \\
71 & 0.005165 & 171 & 0.000517 \\
81 & 0.010286 & 181 & 0.001085 \\
91 & 0.002602 & 191 & 0.000607 \\
\hline
\end{tabular}

In accordance with the real operational conditions, the issue of the set-point tracking is discussed as follows. The tracking target sets in $\delta$ are $R_{\delta}=0.75, t \geq 6$. The results of the tracking issue are shown in Figure 5. From the figure, we notice that the system has successfully tracked the target and subsequently stabilized the target in a period under the action of the NMPC method.

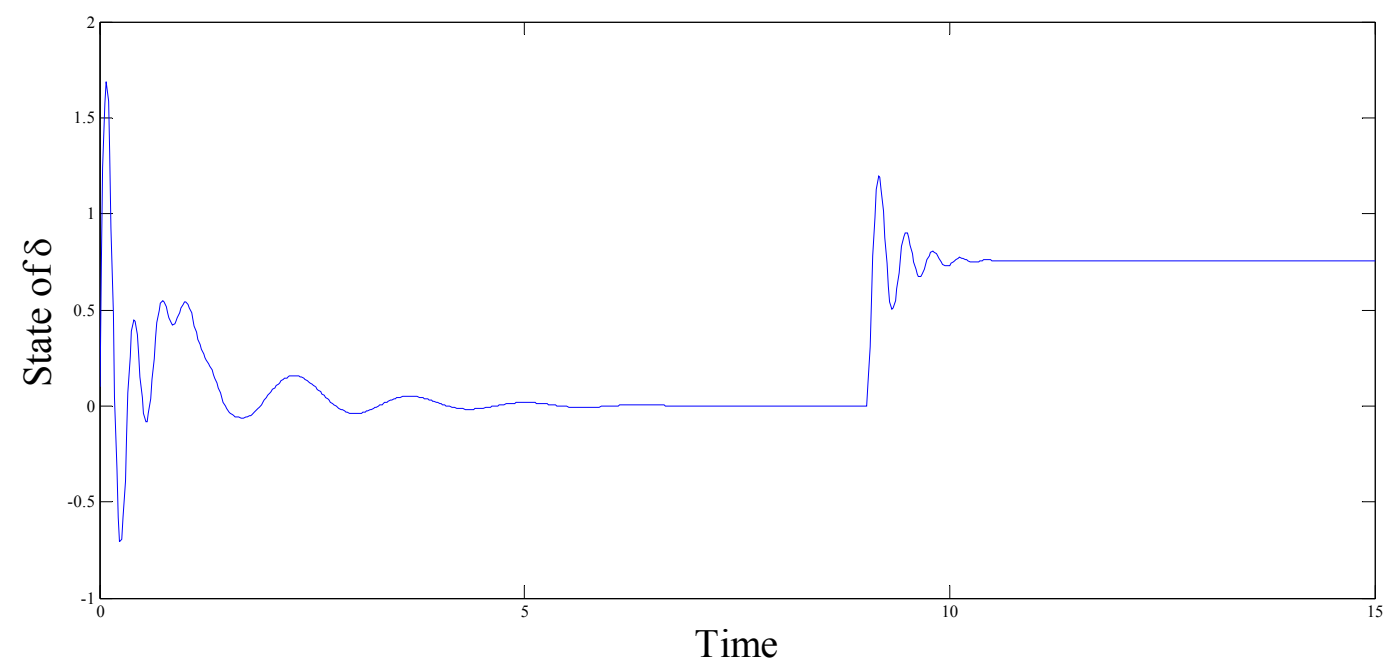

Figure 5. Response of $\delta$ of the system controlled by NMPC method to track the set-point.

In several situations, people cannot access the accurate values of the real plant system. In this case, the model-plant mismatch should be considered to verify the performance of the NMPC method in that situation. The results to verify the stability of the proposed method with the mismatches $d_{\delta}=0.05$, $d_{\omega}=0.1$ and $d_{y}=0.02$ are shown in Figure 6. 


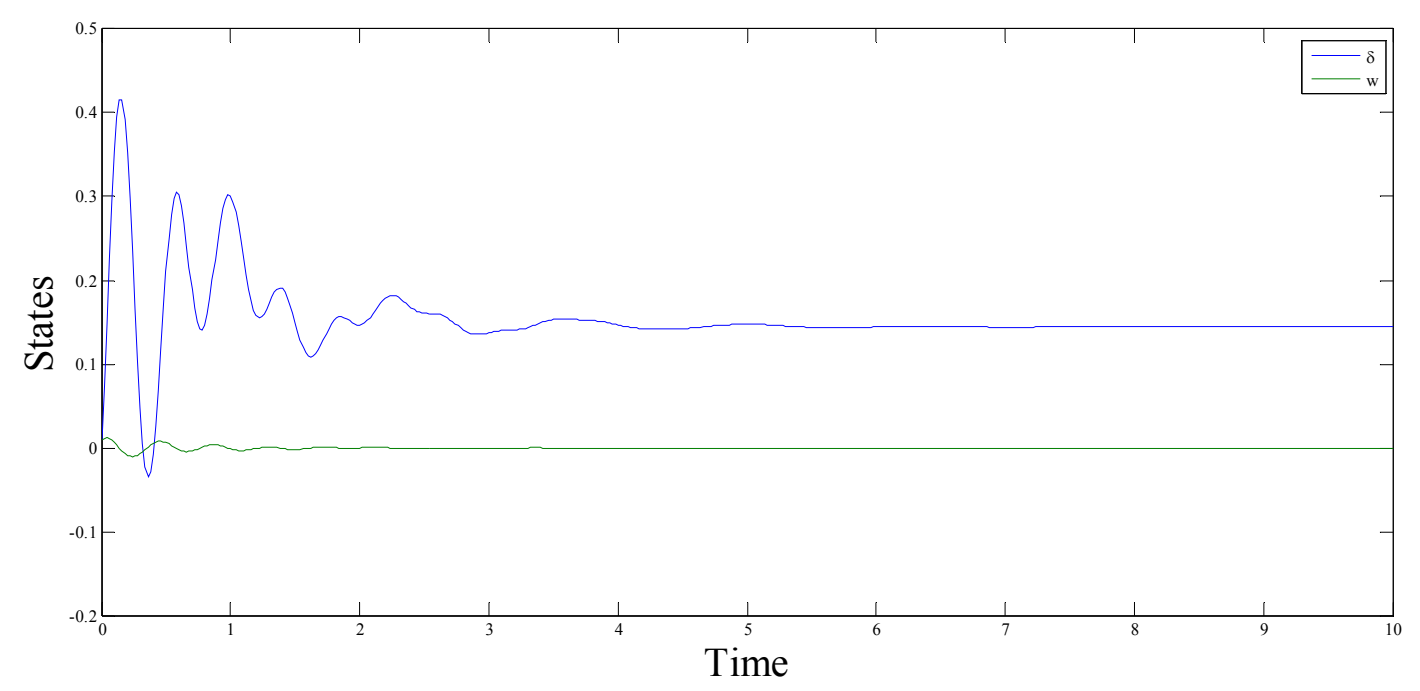

Figure 6. Results of the hydropower station system controlled by NMPC with model-plant mismatch.

Figure 6 shows that the system is still stable with the NMPC controller when there are mismatches existing in the model. The rotor angle $\delta$ fails to reach its equilibrium point, whereas it still operates in the stable state. Therefore, although there is a tiny gap between the equilibrium point and the stability state, the system does operate stably. A disturbance is considered here to illustrate the effectiveness of the proposed method when disturbances exist in the system. The disturbance in the numerical experiments exists in $\delta$, which is $\Delta \delta=1,10 \leq t \leq 12.34$. The response of the system in such a disturbance is shown in Figure 7.

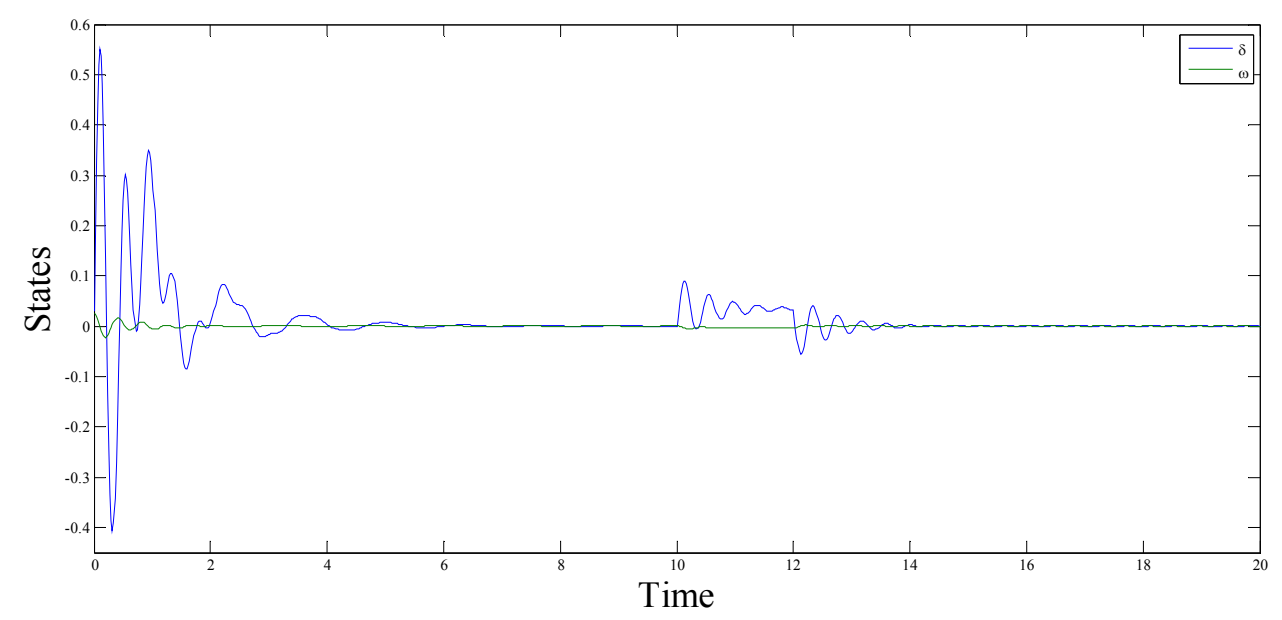

Figure 7. The response of the system with the disturbance $\Delta \delta$ controlled by NMPC method.

From Figure 7, after a period, the system reaches its steady state, pointing out that the NMPC method enjoys the capacity to make the disturbed system stable. As we know, in a real system, randomness exists in some parts caused by the natural features such as the unpredictable water flow and so on. These unpredictable parts can dramatically damage the system. In this case, the stochastic factors should not be ignored in a control system. The following numerical experiment shows the effectiveness of the NMPC method when there are stochastic factors existing in the system. The results are shown in Figure 8. The stochastic factor is added in the rotor angle $\delta$. 


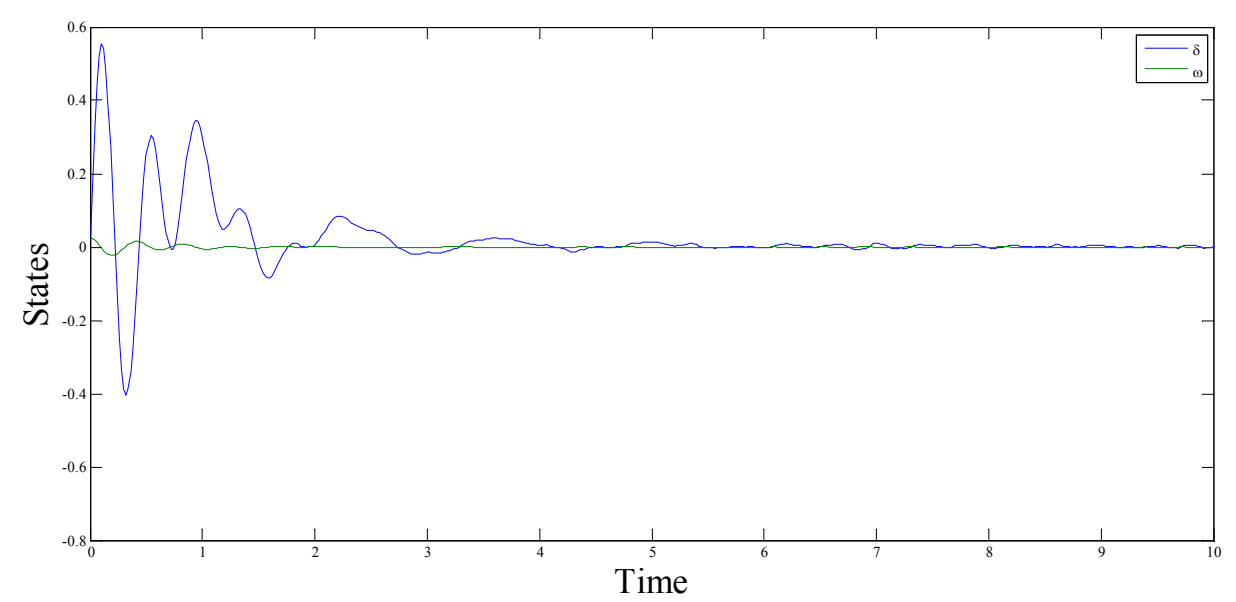

Figure 8. The response of the system with the stochastic factor controlled by the NMPC method.

In Figure 8, the system reaches its steady state in the end. Although, the system spends more time reaching the steady state than the system with the stochastic factor, it is the NMPC method that stabilizes the system to the steady state. That shows the robustness of the NMPC method.

\section{Discussion and Conclusions}

\subsection{Discussion}

We will further discuss NMPC theory and its applications in other engineering fields in this part. Not only can the NMPC method stabilize the hydropower system introduced in this paper, but it can also be applied in other systems described by state-space equations. More importantly, this control method enjoys the capacity to cooperate with other sophisticated control methods. Those control methods have been used in a variety of fields for many years because of its simple model and the experience. Cooperating with those well-developed methods, the mixed predictive control method is able to take advantages of both sides.

\section{Remark 1}

The NMPC controllers enjoy a great advantage in controlling a nonlinear system compared with other traditional control methods such as PID method and so on. The NMPC controllers make it possible for us to control a nonlinear system to the expect states in a short time with less overshoot, which cannot be achieved by PID controllers. In order to illustrate the effectiveness of the NMPC and PID methods, we introduce the PID controller in the system (38) as:

$$
\left\{\begin{array}{l}
\dot{x}_{1}=x_{2} \\
\dot{x}_{2}=x_{3} \\
\dot{x}_{3}=-a_{0} x_{1}-a_{1} x_{2}-a_{2} x_{3}+y \\
\dot{\delta}=\omega_{0} \omega \\
\dot{\omega}=\frac{1}{T_{a b}}\left[m_{t}-\frac{E_{q}^{\prime} V_{s}}{x_{d \Sigma}^{\prime}} \sin \delta-\frac{V_{s}^{2}}{2} \frac{x_{d \Sigma}^{\prime}-x_{q \Sigma}}{x_{d \Sigma}^{\prime} x_{q \Sigma}} \sin 2 \delta-D \omega\right] \\
\dot{y}=\frac{1}{T_{y}}\left(-k_{p}(r-\omega)-\frac{k_{i}}{\omega_{0}} \delta-k_{d} \dot{\omega}-y\right)
\end{array},\right.
$$


where $k_{p}, k_{i}$ and $k_{d}$ are PID parameters, which are $k_{p}=2.0, k_{i}=1.0$, and $k_{d}=2.0$, respectively [36]. PID controller was introduced in state $\omega$.

In order to clearly show the difference between NMPC and PID, we define the index $E$ as:

$$
E=\sqrt{\sum_{i=1}^{n}\left(y_{0 i}-x_{i}\right)^{2}}
$$

The numerical experiments of NMPC and PID are shown in Figure 9.

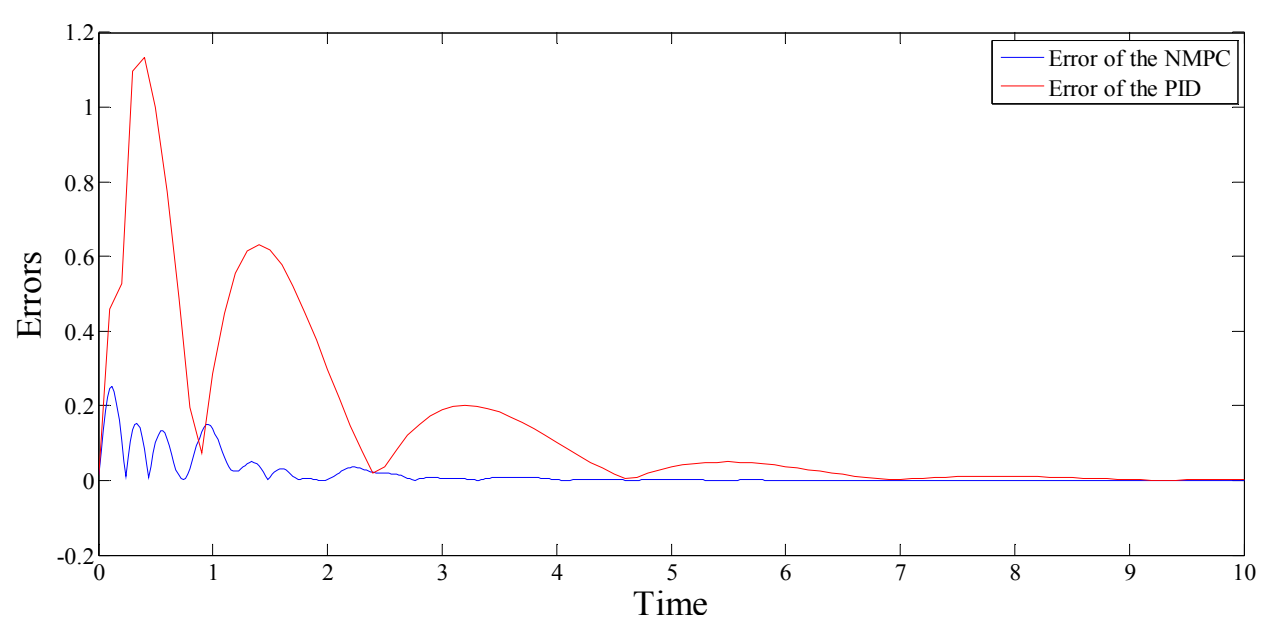

Figure 9. Errors of the NMPC and PID controllers.

From Figure 9, the PID controller stabilizes the nonlinear system (43) slower than NMPC controllers do. Thus, NMPC method has the better capacity to handle a nonlinear system in a short time. It is also shown in Figure 9 that the errors between the expected states and the real states of PID method are larger than that of NMPC, meaning that NMPC method enjoys an excellent performance in control of the nonlinear hydropower system.

\section{Remark 2}

There are several power plants using a simple linear model in control system. The proposed method, of course, is also effective for a linear system. The state-space equations of linear system is:

$$
\dot{\mathbf{x}}=A \mathbf{x}+B \mathbf{u}
$$

where $\mathbf{u}$ are predictive controllers, $A$ is a $n \times n$ matrix and $B$ is $n \times m$ matrix.

In order to apply the predictive control method, we need to substitute Equation (37) for the system (1), and discretize the system (45) to iterate. In the process of calculating the terminal penalty matrix $P$, there is no need to linearize the system through the Jacobian method. One can directly use the matrixes $A$ and $B$ to solve the Equation (11). The linear predictive controller can be obtained from the discretized system (45).

\section{Remark 3}

In Section 4, we suppose the system should be controlled at the origin point, but in many other systems, the system is always stable in a given state $\mathbf{y}_{0}$. In this case, the system Equation (1) is: 


$$
\left\{\begin{array}{l}
\dot{\mathbf{x}}=\mathbf{F}(\mathbf{x}, \mathbf{u}) \\
\mathbf{y}=C \mathbf{x}-\mathbf{y}_{0}
\end{array}\right.
$$

where $\mathbf{y}_{0} \in R^{l}$ are the expect outputs of the system, $\mathbf{y} \in R^{l}$ are the outputs of the system, and $C$ is a $l \times n$ matrix.

After discretizing the system (46), we should substitute the discretized $y$ for the discretized $\mathbf{x}$ in Equation (5), Equation (25) and their sub-equations to control the system to expected states $\mathbf{y}_{0}$. It is to be observed that this is also true for a linear system. Under the circumstance, Equation (46) is:

$$
\left\{\begin{array}{l}
\dot{\mathbf{x}}=A \mathbf{x}+B \mathbf{u} \\
\mathbf{y}=C \mathbf{x}-\mathbf{y}_{0}
\end{array},\right.
$$

and in accordance with Remark 1, system (47) can be controlled to the expected states $\mathbf{y}_{0}$.

\section{Remark 4}

For Remark 1, we know that the NMPC method enjoys a great number of advantages compared to the PID method, especially in a nonlinear system. However, these traditional control methods have been used for a long time. People have built a great body of knowledge about those control methods. For example, PID controllers prevail in almost all the fields, because of their simple structure, stability, convenience and feasibility to set the control parameters. In this case, although NMPC has advantages over the traditional methods, they are not replacing those tradition control methods dramatically. Seldom are the advanced control methods used in practical systems because of the little experience about how to launch such an advanced control method in practice. In addition, the parameters in the advanced control method are more difficult to select than traditional methods. Therefore, we should rationally combine the advantages of the PID with the advantages of other state-of-the-art control methods, for example the NMPC method in this paper. Fortunately, it is possible for us to combine those two control methods.

Take the combination of PID and NMPC methods for example. In order to provide a general method to combine the NMPC method with other control methods, we will generally select PID parameters as the predictive controllers, which means that, in each step, the predictive controller will calculate appropriate PID parameters for the system. We introduce the PID controllers in a nonlinear system (1) as:

$$
\left\{\begin{array}{l}
\dot{\mathbf{x}}=\mathbf{F}(\mathbf{x}, \mathbf{u}) \\
\mathbf{u}=K_{p} \dot{\boldsymbol{\varepsilon}}+K_{i} \boldsymbol{\varepsilon}+K_{d} \ddot{\boldsymbol{\varepsilon}}
\end{array}\right.
$$

where $\boldsymbol{\varepsilon} \in R^{l}$ and $\dot{\boldsymbol{\varepsilon}}=C \mathbf{x}-\mathbf{y}_{0}$ are the errors of the system, respectively; $K_{p} \in R^{l}, \quad K_{i} \in R^{l}$ and $K_{d} \in R^{l}$ are PID parameters, respectively. In this case, we have:

$$
\left\{\begin{array}{l}
\boldsymbol{\varepsilon}=\int_{0}^{\infty}\left(C \mathbf{x}-\mathbf{y}_{0}\right) d t \\
\dot{\boldsymbol{\varepsilon}}=C \mathbf{x}-\mathbf{y}_{0} \\
\ddot{\boldsymbol{\varepsilon}}=C \dot{\mathbf{x}}
\end{array} .\right.
$$

Substituting Equation (49) into Equation (48), the PID controlled system is: 


$$
\left\{\begin{array}{l}
\dot{\mathbf{x}}=\mathbf{F}\left(\mathbf{x}, K_{p}\left(C \mathbf{x}-\mathbf{y}_{0}\right)+K_{i} \boldsymbol{\varepsilon}+K_{d} C \dot{\mathbf{x}}\right) \\
\dot{\boldsymbol{\varepsilon}}=C \mathbf{x}-\mathbf{y}_{0}
\end{array}\right.
$$

Then, the PID parameters are selected as NMPC controllers calculated based on the method proposed in this paper. Subsequently, we can iterate the discretized Equation (50) and apply the predictive control method to Equation (50). Undoubtedly, as we mentioned in Remark 2, this strategy can also be applied in a linear system. What we should do is just to introduce a linear model in Equation (48) shown as:

$$
\left\{\begin{array}{l}
\dot{\mathbf{x}}=A \mathbf{x}+B \mathbf{u} \\
\mathbf{u}=K_{p} \dot{\boldsymbol{\varepsilon}}+K_{i} \boldsymbol{\varepsilon}+K_{d} \ddot{\boldsymbol{\varepsilon}}
\end{array}\right.
$$

Substituting Equation (49) into Equation (51), the PID controlled system of a linear system is:

$$
\left\{\begin{array}{l}
\dot{\mathbf{x}}=A \mathbf{x}+B\left(K_{p}\left(C \mathbf{x}-\mathbf{y}_{0}\right)+K_{i} \boldsymbol{\varepsilon}+K_{d} C \dot{\mathbf{x}}\right) \\
\dot{\boldsymbol{\varepsilon}}=C \mathbf{x}-\mathbf{y}_{0}
\end{array} .\right.
$$

System (52) can be controlled based on the NMPC method by selecting the PID parameters as the predictive controllers, which means the parameters will be calculated in each step to control the system (45).

Ae numerical experiment demonstrating the proposed combination method is presented here. The system of the numerical experiment is Equation (43). The results are shown in Figure 10. In the numerical experiment, the PID parameters were decided through NMPC method. This has a great advantage. As aforementioned, there are large numbers of PID-based control systems. For improving these systems, people just need to change the method to determine the PID parameters, which is more convenient and economical than replacing the whole system.

From Figure 10, we know that the system is stable, pointing out the efficiency of the method. This is just an example of the combination of PID method and NMPC method or MPC method. Practically, people can combine any given controlled system with the NMPC method or MPC method like this, only if the system can be described as Equation (1) or Equation (45).

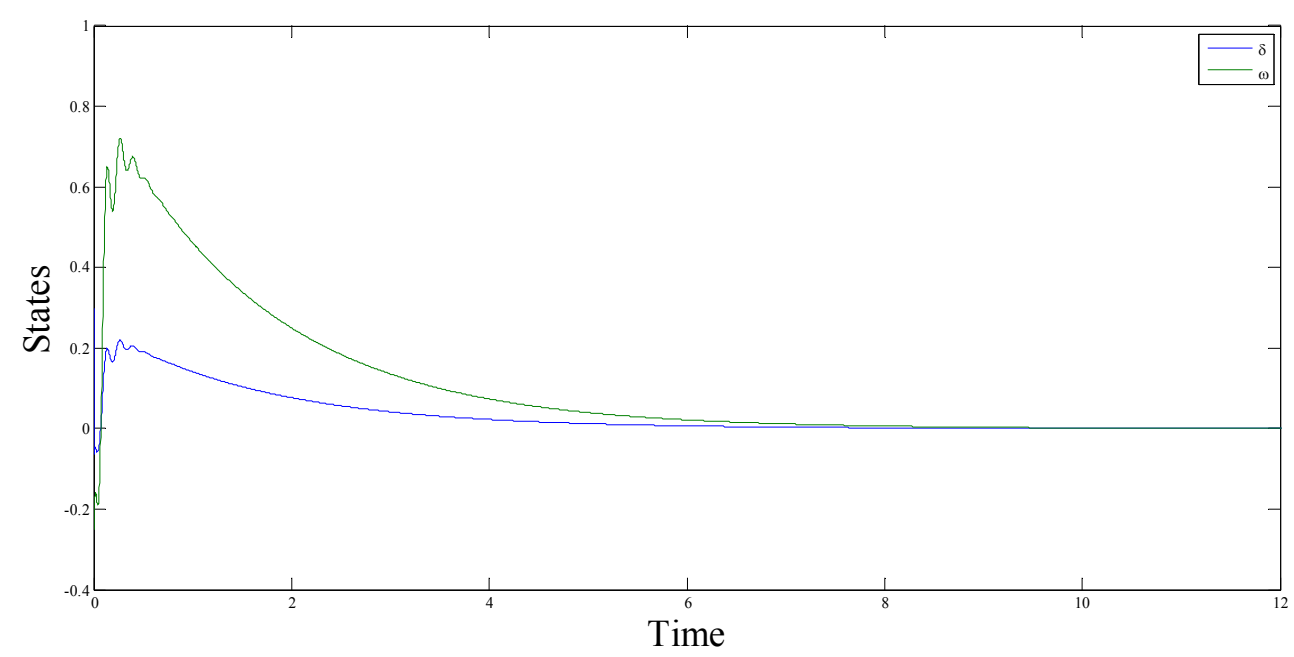

Figure 10. The response of the hydropower station system with PID combined NMPC method. 


\subsection{Conclusions}

In this paper, we propose a simple method to select an appropriate performance index to control a system based on the nonlinear predictive control method. The method is through the use of the Lyapunov equation to select a terminal penalty function, and its stability is proved by the Lyapunov function. Subsequently, a nonlinear hydropower plant system is controlled by the method. The six-dimensional model is complex enough to describe a hydropower system accurately. In the hydropower system, the hydro-turbine system, the penstock system, the generator system, and the hydraulic servo system are considered. Eventually, not only do we compare NMPC method with the traditional PID method, but we also provide strategies to apply the NMPC method to a linear system, and to combine NMPC method with other traditional control methods. It is worth mentioning that the paper not just provides an example of the NMPC method, but offers a general way to use the NMPC method in different situations.

\section{Acknowledgments}

This work was supported by the scientific research foundation of National Science Foundation (51479173, 51279167), Fundamental Research Funds for the Central Universities (201304030577), Scientific research funds of Northwest A\&F University (2013BSJJ095), the scientific research foundation on water engineering of Shaanxi Province (2013slkj-12) and the Science Fund for Excellent Young Scholars from Northwest A\&F University.

\section{Author Contributions}

Diyi Chen and Runfan Zhang conceived and designed the experiments; Runfan Zhang performed the experiments; Diyi Chen and Xiaoyi Ma analyzed the data; Runfan Zhang wrote the paper.

\section{Conflicts of Interest}

The authors declare no conflict of interest.

\section{References}

1. Edward, G.R.; Xu, J.C. Mekong hydropower development. Science 2011, 332, 178-179.

2. Mahmoud, M.; Dutton, K.; Denman, M. Design and simulation of a nonlinear fuzzy controller for a hydropower plant. Electr. Power Syst. Res. 2005, 73, 87-99.

3. Li, C.L.; Zhou, J.Z.; Ouyang, S.; Ding, X.L.; Chen, L. Improved decomposition-coordination and discrete differential dynamic programming for optimization of large-scale hydropower system. Energ. Convers. Manag. 2014, 84, 363-373.

4. Martínez-Lucas, G.; Sarasúa, J.I.; Sánchez-Fernández, J.Á.; Wilhelmi, J.R. Power-frequency control of hydropower plants with long penstocks in isolated systems with wind generation. Renew. Energy 2015, 83, 245-255.

5. Márquez, J.L.; Molina, M.G.; Pacas, J.M. Dynamic modeling, simulation and control design of an advanced micro-hydro power plant for distributed generation applications. Int. J. Hydrog. Energy 2010, 35, 5772-5777. 
6. Mahmoud, M.; Dutton, K.; Denman, M. Dynamical modelling and simulation of a cascaded reserevoirs hydropower plant. Electr. Power Syst. Res. 2004, 70, 129-139.

7. Zoby, M.R.G.; Yanagihara, J.I. Primary control system and stability analysis of a hydropower plant. In Power Plants and Power Systems Control 2006; Westwick, D., Ed.; Elsevier: Kidlington, UK; Burlington, MA, USA, 2007; pp. 165-170.

8. Ren, M.; Wu, D.; Zhang, J.; Jiang, M. Minimum entropy-based cascade control for governing hydroelectric turbines. Entropy 2014, 16, 3136-3148.

9. Ying, H. Theory and application of a novel fuzzy PID controller using a simplified Takagi-Sugeno rule scheme. Inf. Sci. 2000, 123, 281-293.

10. Clarke, D.W. Adaptive predictive control. Annu. Rev. Control 1996, 20, 83-94.

11. Miller, D.E. A new approach to adaptive control: No nonlinearities. Syst. Control Lett. 2003, 49, 67-79.

12. Mei, R.; Chen, M.; Guo, W.W. Robust adaptive control scheme for optical tracking telescopes with unknown disturbances. Opt. Int. J. Light Electron Opt. 2015, 126, 1185-1190.

13. Ren, M.; Zhang, J.; Jiang, M.; Tian, Y.; Hou, G. Statistical information based single neuron adaptive control for non-gaussian stochastic systems. Entropy 2012, 14, 1154-1164.

14. Hu, B.; Michel, A.N. Robustness analysis of digital feedback control systems with time-varying sampling periods. J. Franklin Inst. 2000, 337, 117-130.

15. Stich, M.; Beta, C. Control of pattern formation by time-delay feedback with global and local contributions. Physica D 2010, 239, 1681-1691.

16. Xin, B.G.; Wu, Z.H. Projective synchronization of chaotic discrete dynamical systems via linear state error feedback control. Entropy 2015, 17, 2677-2687.

17. Bidarvatan, M.; Shahbakhti, M.; Jazayeri, S.A.; Koch, C.R. Cycle-to-cycle modeling and sliding mode control of blended-fuel HCCI engine. Control Eng. Pract. 2014, 24, 79-91.

18. Tian, X.M.; Fei, S.M. Robust control of a class of uncertain fractional-order chaotic systems with input nonlinearity via an adaptive sliding mode technique. Entropy 2014, 16, 729-746.

19. Yashar, T.; Wang, J.D. Chaos control and synchronization of a hyperchaotic Zhou system by integral sliding mode control. Entropy 2014, 16, 6539-6552.

20. Chen, D.Y.; Zhao, W.L.; Ma, X.Y.; Zhang, R.F. No-chattering sliding mode control chaos in Hindmarsh-Rose neurons with uncertain parameters. Comput. Math. Appl. 2011, 61, 3161-3171.

21. Long, L.J.; Zhao, J. Adaptive fuzzy tracking control of switched uncertain nonlinear systems with unstable. Fuzzy Sets Syst. 2015, 273, 49-67.

22. Huang, H.; Chen, L.; Hu, E. A neural network-based multi-zone modelling approach for predictive control system design in commercial buildings. Energy Build. 2015, 97, 86-97.

23. Chen, H.Y.; Liu, M.Q.; Zhang, S.L. Robust $H_{\infty}$ finite-time control for discrete Markovian jump systems with disturbances of probabilistic distributions. Entropy 2015, 17, 346-367.

24. Grüne, L.; Pannek, J. Nonlinear Model Predictive Control; Springer: London, UK, 2011.

25. Chen, H.; Allgower, F. A quasi-infnite horizon nonlinear model predictive control scheme with guaranteed stability. Automatica 1998, 34, 1205-1217.

26. Chen, C.C.; Shaw, L. On receding horizon feedback control. Automatica 1982, 18, 349-352.

27. Mayne, D.Q. Model predictive control: Recent developments and future promise. Automatica 2014, 50, 2967-2986. 
28. Xu, J.; Huang, X.L.; Mu, X.M.; Wang, S.L. Model predictive control based on adaptive hinging hyperplanes mode. J. Process Control 2012, 22, 1821-1831.

29. Martí, R.; Lucia, S.; Sarabia, D.; Paulen, R.; Engell, S.; de Prada, C. Improving scenario decomposition algorithms for robust nonlinear model predictive control. Comput. Chem. Eng. 2015, $79,30-45$.

30. Zeng, X.H.; Yang, N.N.; Wang, J.N.; Song, D.F.; Zhang, N.; Shang, M.L.; Liu, J.X. Predictive-model-based dynamic coordination control strategy for power-split hybrid electric bus. Mech. Syst. Signal Process. 2015, 60, 785-798.

31. Li, S.Y.; Zhang, Y.; Zhu, Q.M. Nash-optimization enhanced distributed model predictive control applied to the Shell benchmark problem. Inf. Sci. 2005, 170, 329-349.

32. Yang, J.; Li, X.; Mou, H.G.; Jian, L. Predictive control of solid oxide fuel cell based on an improved Takagi-Sugeno fuzzy model. J. Power Sources 2009, 93, 699-705.

33. Roubos, J.A.; Mollov, S.; Babuska, R.; Verbruggen, H.B. Fuzzy model-based predictive control using Takagi-Sugeno models. Int. J. Approx. Reason. 1999, 22, 3-30.

34. Sarimveis, H.; Bafas, G. Fuzzy model predictive control of non-linear processes using genetic algorithms. Fuzzy Sets Syst. 2003, 139, 59-80.

35. Jiang, Z.P.; Wang, Y. Input-to-state stability for discrete time nonlinear systems. Automatica 2001, 37, 875-867.

36. Xu, B.B.; Chen, D.Y.; Zhang, H.; Wang, F.F. Modeling and stability analysis of a fractional-order Francis hydro-turbine governing system. Chaos Soliton. Fract. 2015, 75, 50-61.

37. Shen, Z.Y. Hydraulic Turbine Reglation, 3rd ed.; China Water Press: Beijing, China, 1998. (In Chinese)

38. Chen, D.Y.; Ding, C.; Do, Y.H.; Ma, X.Y. Zhao, H.; Wang, Y.C. Nonlinear dynamic analysis for a Francis hydro-turbine governing system and its control. J. Franklin Inst. 2014, 351, 4596-4618.

(C) 2015 by the authors; licensee MDPI, Basel, Switzerland. This article is an open access article distributed under the terms and conditions of the Creative Commons Attribution license (http://creativecommons.org/licenses/by/4.0/). 